\title{
Language-specific memory for everyday arithmetic facts in Chinese-English bilinguals
}

\author{
Yalin Chen $^{1} \cdot$ Jill Yanke $^{1}$ - Jamie I. D. Campbell ${ }^{1}$
}

Published online: 12 August 2015

(C) Psychonomic Society, Inc. 2015

\begin{abstract}
The role of language in memory for arithmetic facts remains controversial. Here, we examined transfer of memory training for evidence that bilinguals may acquire languagespecific memory stores for everyday arithmetic facts. Chinese-English bilingual adults $(n=32)$ were trained on different subsets of simple addition and multiplication problems. Each operation was trained in one language or the other. The subsequent test phase included all problems with addition and multiplication alternating across trials in two blocks, one in each language. Averaging over training language, the response time (RT) gains for trained problems relative to untrained problems were greater in the trained language than in the untrained language. Subsequent analysis showed that English training produced larger RT gains for trained problems relative to untrained problems in English at test relative to the untrained Chinese language. In contrast, there was no evidence with Chinese training that problem-specific RT gains differed between Chinese and the untrained English language. We propose that training in Chinese promoted a translation strategy for English arithmetic (particularly multiplication) that produced strong cross-language generalization of practice, whereas training in English strengthened relatively weak, English-language arithmetic memories and produced little generalization to Chinese (i.e., English training did not induce an English translation strategy for Chinese language trials).
\end{abstract}

Jamie I. D. Campbell

jamie.campbell@usask.ca

Yalin Chen

yalin.chen@usask.ca

1 Department of Psychology, University of Saskatchewan, 9 Campus Drive, Saskatoon, SK, Canada S7N 5A5
The results support the existence of language-specific strengthening of memory for everyday arithmetic facts.

Keywords Language $\cdot$ Bilingual arithmetic . Transfer of training

There is diverse behavioral (Campbell \& Epp, 2004; Pica, Lemer, Izard, \& Dehaene, 2004), developmental (Venkatraman, Siong, Chee, \& Ansari, 2006), and neurological evidence (Cohen, Dehaene, Chochon, Lehericy, \& Naccache, 2000; Dehaene, Spelke, Pinal, Stanescu, \& Tsivkin, 1999; Salillas \& Carreiras, 2014) that language plays an important role in the development and performance of arithmetic skills. Nonetheless, whether or not language is essential for cognitive arithmetic remains controversial (Butterworth \& Reeve, 2008; Butterworth, Reeve, \& Reynolds, 2011; Everett \& Madora, 2012; Frank, Everett, Fedorenko, \& Gibson, 2008; Spaepen, Coppola, Spelke, Carey, \& Goldin-Meadow, 2011). One important source of behavioral evidence has come from the study of arithmetic memory in bilinguals (see Rusconi, Galfano, \& Job, 2007, for a recent review). Dehaene et al. (1999; see also Pica et al., 2004; Spelke \& Tsivkin, 2001) presented evidence that language-based processes mediate exact arithmetic, whereas a language-independent magnitude representation mediates approximate arithmetic. In the Dehaene et al. study, RussianEnglish bilinguals were trained on sets of exact and approximate multi-digit arithmetic problems in their two languages. At test, exact calculations showed large response time (RT) costs of language switching and poor generalization to novel problems for both languages. In contrast, approximate arithmetic showed language independence at test, and training generalized to new problems without loss. Dehaene et al. concluded that training exact arithmetic was language specific, whereas approximate 
arithmetic depended upon a language-independent quantity representation (but see Gelman \& Butterworth, 2005).

Such language-specific transfer of learning demonstrates that newly-learned arithmetic facts may be at least temporarily stored in the language in which they are learned, but does this generalize to adults' memory for everyday arithmetic facts such as $2+3$ and $4 \times 5$ ? Campbell and Epp (2004) proposed that Chinese-English bilinguals with extensive experience in both languages may possess memory networks of simple addition and multiplication facts in each language. To pursue this, Campbell and Dowd (2012) examined retrieval-induced interference in Chinese-English bilinguals' addition-fact memory that is induced by retrieval practice of multiplication counterparts. Participants practiced multiplication problems (e.g., $6 \times 7=$ ?), answering a subset in Chinese and another subset in English. Then separate groups answered corresponding addition problems $(6+7=$ ?) and control addition problems in either Chinese or English. The results reported by Campbell and Dowd, and subsequent analyses by Campbell and Therriault (2013), demonstrated RT costs for addition problems after their multiplication counterparts were practiced in the same language relative to practice in the other language. Thus, the Campbell and Dowd experiment provided evidence of language-specific retrieval-induced interference in Chinese-English bilinguals' memory for addition facts.

The theory that Chinese-English bilinguals may acquire memory networks for arithmetic facts in both languages makes another clear, but as yet untested, prediction. Facilitative transfer of learning from practicing a given problem should be language specific. As described before, Dehaene et al. (1999) demonstrated this in bilingual adults memorizing new, unfamiliar multi-digit arithmetic facts, but it has never been demonstrated in a bilingual individual's memory for everyday arithmetic facts. Here a group of Chinese-English bilingual adults $(n=$ 32) were trained on different subsets of simple addition and multiplication problems involving all combinations of the numbers two through nine. Each operation was trained in one language or the other. The subsequent test phase included all the problems with addition and multiplication alternating across trials in two blocks, one in each language. If performance is based on language-specific memory representations then there should be little problem-specific transfer of training for problems practiced in one language and tested in the other, compared to the facilitation observed when training and testing of a problem occurred in the same language.

\section{Method}

\section{Participants}

Thirty-two Chinese-English bilinguals (21 females, aged 1729 years, mean $=22.4, \mathrm{SD}=2.8,30$ right-handed) were recruited via on-line advertising at the University of Saskatchewan and received CA $\$ 10$ for participation. Their first language for arithmetic was Chinese, and English language experience varied from 8 to 21 years $($ mean $=13.0$, $\mathrm{SD}=3.02)$.

\section{Apparatus}

Stimuli appeared on two monitors using E-prime 2.0 (Psychology Software Tools, Inc.) with one monitor viewed by the participant and the other by the experimenter. The participant sat approximately $50 \mathrm{~cm}$ from their monitor holding a microphone connected to the computer through Eprime's SRbox. Sound input through the microphone provided the stop-signal to a software clock used to measure RT.

\section{Stimuli}

Addition and multiplication problems were made from the 36 possible pairings of the numbers two through nine. The 36 pairs were divided into two sets of 18 with the numbers two through nine appearing in four pairs in each set. Set 1 included $22,25,34,26,35,44,36,29,47,39,48,57,58,67,68,77,89$, and 99. Set 2 included 23, 24, 33, 27, 45, 28, 37, 46, 55, 38, 56, 66, 49, 59, 69, 78, 79, and 88. On English language trials, problems were lower-case English number words separated by the operation sign $(+$ or $\times)$ with adjacent spaces (e.g., the pair 58 appeared as five $\times$ eight or five + eight). On Chinese language trials, Chinese numbers were used for the operands (e.g., 五 $\times$ 八 or 五 + 八). Problems were displayed horizontally in 14-point black Courier New against a white background and appeared with the smaller number on the left when the two operands differed.

\section{Design}

Sixteen participants were assigned to each of two training conditions. One group trained addition in Chinese and multiplication in English and the other group trained addition in English and multiplication in Chinese. Thus, all four Operation $\times$ Language training combinations were represented by the two training groups. There were eight training blocks with operation, training language, and problem set switching across successive blocks. For example, a participant who trained addition in English using the Set 1 operands in Block 1 switched in Block 2 to training multiplication in Chinese using the Set 2 operands and continued to alternate between these two configurations across the eight training blocks. The Operation $\times$ Language assignment (i.e., English addition and Chinese multiplication or Chinese addition and English multiplication), the operand set assigned to each operation (Set 1 or 2), and the operation for the first training block were counterbalanced across sets of eight participants. 
The test phase had two blocks of 72 trials. One test block was in Chinese and the other in English. The language for the first test block was counterbalanced with the factors counterbalanced in the training phase (see preceding paragraph). As such, complete counterbalancing was achieved over groups of 16 participants. In each test block, trials alternated between addition and multiplication problems with all 18 Set 1 and 18 Set 2 operand pairs used for each operation. Problem order for each operation was random for each participant except that problems involving the same operand pair (e.g., $4 \times 6$ and $4+6$ ) were separated by at least 20 trials.

\section{Procedure}

The 45-min session occurred in a quiet room with the first author present. Participants were asked to answer problems quickly and accurately. Each trial began with a central fixation dot that appeared for $1 \mathrm{~s}$, disappeared for $.5 \mathrm{~s}$, and then flashed on and off twice over a 1-s interval. The problem then appeared with the operation sign at fixation. Timing began synchronized with the problem display and terminated with the participant's verbal response. The experimenter typed in the given answer and then the fixation dot for the next trial appeared. Participants were offered a brief break between trial blocks.

\section{Results}

\section{Training phase}

A total of $2.1 \%$ of training RTs were discarded as outliers more than 3 SD from a participant's mean RT for each Operation $\times$ Language $\times$ Block cell. The mean rate of arithmetic errors (i.e., incorrect answers) during training was 3.7 $\%$. Figure 1 presents the mean RT for correct trials by operation, language, and block. Table 1 contains the corresponding mean percentage of errors committed. As error rates were low and there was no evidence of a speed-accuracy trade off, the analyses focused on RT.

Mean RT for correct trials was analysed in an ANOVA with group (trained addition in English and multiplication in Chinese, or trained addition in Chinese and multiplication in English) as a between-participants factor, and operation (addition, multiplication) and training blocks (1 to 4 for each Operation $\times$ Language combination) as repeated-measures factors. Overall, multiplication was slower on average (1, $305 \mathrm{~ms})$ than addition $(1,181 \mathrm{~ms})[F(1,30)=9.2, \mathrm{MSE}=$ $\left.106,362, \eta p^{2}=.24, p=.005\right]$, which is the usual finding (e.g., Campbell \& Xue, 2001). Both operations were performed slower in English than in Chinese $(+576 \mathrm{~ms}$ for addition and $+517 \mathrm{~ms}$ for multiplication), as indicated by the Group $\times$ Operation interaction $[F(1,30)=179.8$, MSE $=$

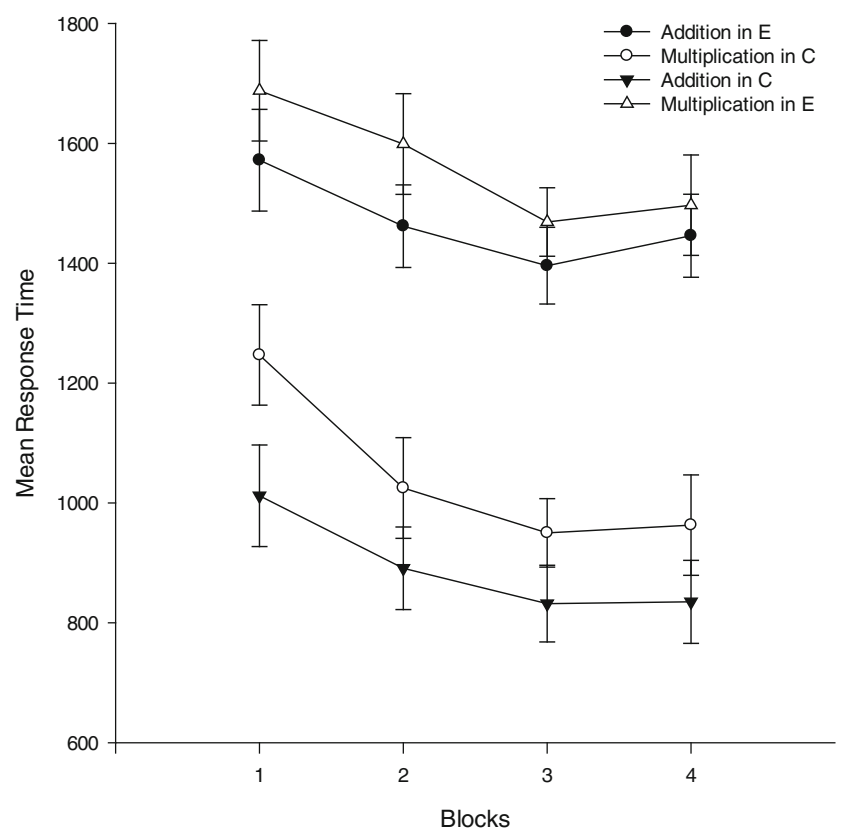

Fig. 1 Mean response time in ms by operation, language, and block in the training phase. $\mathrm{C}=$ training language Chinese. $\mathrm{E}=$ training language English. Error bars are \pm 1 standard error

$\left.106,362, \eta p^{2}=.86, p<.001\right]$. It should be noted that here the Group $\times$ Operation test corresponds to the overall RT advantage for Chinese relative to English because the two groups trained with opposite Language $\times$ Operation mappings. As Fig. 1 shows, participants sped up substantially across blocks $\left[F(3,30)=38.2, \mathrm{MSE}=16,006, \eta^{2}=.56\right.$, $p<.001]$, with the most speed-up occurring during Blocks 1 and 2 and RTs leveling off in Blocks 3 and 4 as participants approached speed-up asymptote for each Operation $\times$ Language combination within the training phase. There were no other significant effects in the training RT analysis (all $p>.23$ ).

\section{Test phase}

A total of $2.1 \%$ of RTs were excluded as outliers more than 3 SD from a participant's mean RT for each Language Type (trained or untrained) $\times$ Operation cell. Table 2 presents mean RT by group, operation, language type (trained or untrained), and problem type (trained or untrained). Table 3 presents the corresponding percentages of errors. The overall error rate during the test phase was $4.6 \%$, but there were too few in many cells for a full statistical analysis; consequently, we focused on analyses of mean RT for correct trials. Our design did not fully cross training language and operation. Averaging over the two Operation $\times$ Language groups, the language factor represents training and test language either the same (trained) or different (untrained). We first report analyses of the test phase RTs averaged over group, and then report analyses using only one between-group factor (i.e., training language or operation), 
Table 1 Mean percentage of errors and standard error (in brackets) in the training phase for training group, operation (addition, multiplication), and block

\begin{tabular}{|c|c|c|c|c|}
\hline & \multicolumn{2}{|c|}{$\begin{array}{l}\text { Trained addition in English and multiplication } \\
\text { in Chinese }\end{array}$} & \multicolumn{2}{|c|}{$\begin{array}{l}\text { Trained addition in Chinese and multiplication } \\
\text { in English }\end{array}$} \\
\hline & Addition & Multiplication & Addition & Multiplication \\
\hline Block 1 & $4.2(1.4)$ & $3.5(1.0)$ & $3.5(1.5)$ & $4.2(1.2)$ \\
\hline Block 2 & $4.9(1.2)$ & $2.8(0.9)$ & $2.1(0.9)$ & $3.5(0.9)$ \\
\hline Block 3 & $4.2(1.3)$ & $3.5(1.1)$ & $2.1(0.9)$ & $4.5(1.0)$ \\
\hline Block 4 & $4.5(1.3)$ & $4.5(1.4)$ & $3.1(1.3)$ & $3.8(1.3)$ \\
\hline
\end{tabular}

which provide more specific information about transfer effects across training language and operation.

The Language Type (trained or untrained) $\times$ Problem Type (trained or untrained) $\times$ Operation repeated-measures ANOVA showed that, as in the training phase, mean RT was slower for multiplication $(1,502 \mathrm{~ms})$ than addition $(1,364 \mathrm{~ms})$ in the test phase $\left[F(1,31)=30.0, \mathrm{MSE}=40,703, \eta \mathrm{p}^{2}=.49\right.$, $p<.001]$. Overall, participants were faster to answer trained $(1,370 \mathrm{~ms})$ than untrained $(1,496 \mathrm{~ms})$ problems $[F(1,31)=$ $\left.71.7, \mathrm{MSE}=14,098, \eta \mathrm{p}^{2}=.70, p<.001\right]$, but the RT gains for trained problems relative to untrained problems were greater in multiplication $(156 \mathrm{~ms})$ than in addition $(95 \mathrm{~ms})[F(1,31)=$ 5.0, MSE $\left.=11,801, \eta p^{2}=.14, p=.032\right]$. Overall, mean RT was not significantly faster in the trained language $(1,404 \mathrm{~ms})$ compared to the untrained language $(1,463 \mathrm{~ms})[F(1,30)=$ $\left.3.2, \operatorname{MSE}=71,181, \eta p^{2}=.09, p=.08\right]$ but, most important, there was a Language Type $\times$ Problem Type interaction $[F(1$, $\left.31)=12.2, \operatorname{MSE}=8,729, \eta p^{2}=.28, p=.001\right]$. This occurred because RT gains for trained problems relative to untrained problems were greater in the trained language $(167 \mathrm{~ms})$ than in the untrained language $(85 \mathrm{~ms})$. This confirms that training resulted in language-specific transfer expressed in RT gains.

In the following analyses, we examined problem-specific RT gains (i.e., mean RT for untrained problems minus the mean RT for trained problems) by operation with training language as a between-group factor and, in separate analyses, examined RT gains by training language with operation as a between-group factor. Figure 2 presents the mean RT gain for each Group $\times$ Operation $\times$ Language Type cell.

\section{Effects of training language on transfer within operation}

Multiplication A Group (Chinese vs. English training for multiplication) $\times$ Language Type (language at test trained vs untrained) of mean RT gains indicated that gains were larger overall in the trained language $(200 \mathrm{~ms})$ than in the untrained language $(112 \mathrm{~ms})\left[F(1,30)=7.1, \mathrm{MSE}=17,491, \eta_{\mathrm{P}}{ }^{2}=0.19\right.$, $p=0.012]$, but the Group $\times$ Language Type interaction $[F(1$, $\left.30)=6.8, \mathrm{MSE}=17,491, \eta_{\mathrm{P}}{ }^{2}=0.19, p=0.014\right]$ indicated that when English was the trained language for multiplication, RT gains were greater in the trained language $[247 \mathrm{~ms}, t(15)=$ $7.11, p<.0001, \mathrm{SE}=34.7$ against 0 gain] compared to the untrained Chinese language [72 ms, $t(15)=5.07, p<.0001$, $\mathrm{SE}=14.3]$. In contrast, when Chinese was the trained language, RT gains were practically equivalent in the trained Chinese language $[154 \mathrm{~ms}, t(15)=5.32, p<.0001, \mathrm{SE}=$ 28.9] and untrained English language [152 $\mathrm{ms}, t(15)=3.16$, $p=.007, \mathrm{SE}=48.0]$. Thus, multiplication training generalized to English when Chinese was trained, but there was relatively little generalization of multiplication practice to Chinese when English was trained.

Table 2 Mean response time (ms) and standard error (in brackets) in the test phase for training group, operation (addition, multiplication), language trained or untrained for this operation, and problem trained or untrained for this operation

\begin{tabular}{|c|c|c|c|c|}
\hline & \multicolumn{2}{|c|}{ Trained addition in English and multiplication in Chinese } & \multicolumn{2}{|c|}{ Trained addition in Chinese and multiplication in English } \\
\hline & Addition & Multiplication & Addition & Multiplication \\
\hline Language trained & $\mathrm{E}$ & $\mathrm{C}$ & $\mathrm{C}$ & $\mathrm{E}$ \\
\hline Problem trained & $1652(147)$ & $1059(59)$ & $955(53)$ & $1615(127)$ \\
\hline Problem untrained & $1827(131)$ & $1212(61)$ & $1046(58)$ & $1862(126)$ \\
\hline Language untrained & $\mathrm{C}$ & $\mathrm{E}$ & $\mathrm{E}$ & $\mathrm{C}$ \\
\hline Problem trained & $1121(83)$ & $2040(138)$ & $1538(87)$ & $983(54)$ \\
\hline Problem untrained & $1172(65)$ & $2192(143)$ & $1603(103)$ & $1055(57)$ \\
\hline
\end{tabular}

Note. $\mathrm{E}=$ test language English, $\mathrm{C}=$ test language Chinese 
Table 3 Mean error rate and standard errors (in brackets) for training group, operation (addition, multiplication), language trained or untrained for this operation, and problem trained or untrained for this operation

\begin{tabular}{|c|c|c|c|c|}
\hline & \multicolumn{2}{|c|}{ Trained addition in English and multiplication in Chinese } & \multicolumn{2}{|c|}{ Trained addition in Chinese and multiplication in English } \\
\hline & Addition & Multiplication & Addition & Multiplication \\
\hline Language trained & $\mathrm{E}$ & $\mathrm{C}$ & $\mathrm{C}$ & $\mathrm{E}$ \\
\hline Problem trained & $2.4(0.9)$ & $1.7(1.0)$ & $0.7(0.5)$ & $3.1(1.1)$ \\
\hline Problem untrained & $3.5(0.9)$ & $3.1(0.9)$ & $7.3(2.2)$ & $6.6(1.4)$ \\
\hline Language untrained & $\mathrm{C}$ & $\mathrm{E}$ & $\mathrm{E}$ & $\mathrm{C}$ \\
\hline Problem trained & $2.4(1.1)$ & $6.6(1.5)$ & $3.8(1.0)$ & $3.8(1.4)$ \\
\hline Problem untrained & $4.2(1.5)$ & $6.6(1.5)$ & $4.9(1.8)$ & $10.4(2.0)$ \\
\hline
\end{tabular}

Note. $\mathrm{E}=$ test language English, $\mathrm{C}=$ test language Chinese

Addition The corresponding analysis of addition RT gains confirmed a main effect of Language Type $[F(1,30)=$ $\left.4.4, \mathrm{MSE}=20,348, \eta_{\mathrm{P}}{ }^{2}=0.13, p=0.044\right]$, whereby RT gains were greater in the trained language $(133 \mathrm{~ms})$ than in the untrained language $(58 \mathrm{~ms})$. Addition presented significant problem-specific RT gains in the trained language both for English [175 ms, $t(15)=2.88, p=.01, \mathrm{SE}=60.7]$ and Chinese [91 ms, $t(15)=3.69, p=.002, \mathrm{SE}=24.7]$. There was little evidence for generalization to the untrained language, however, either for Chinese [51 ms, $t(15)=1.54, p=$ $.14, \mathrm{SE}=32.9]$ or English [65 ms, $t(15)=1.72, p=.11, \mathrm{SE}=$ 37.6]. For addition, the two-way interaction between Group (i.e., training language) and Language Type was not statistically significant ( $p=.18$ ). Thus, unlike multiplication, there was no evidence that RT gains from addition training in either Chinese or English generalized to the other language.

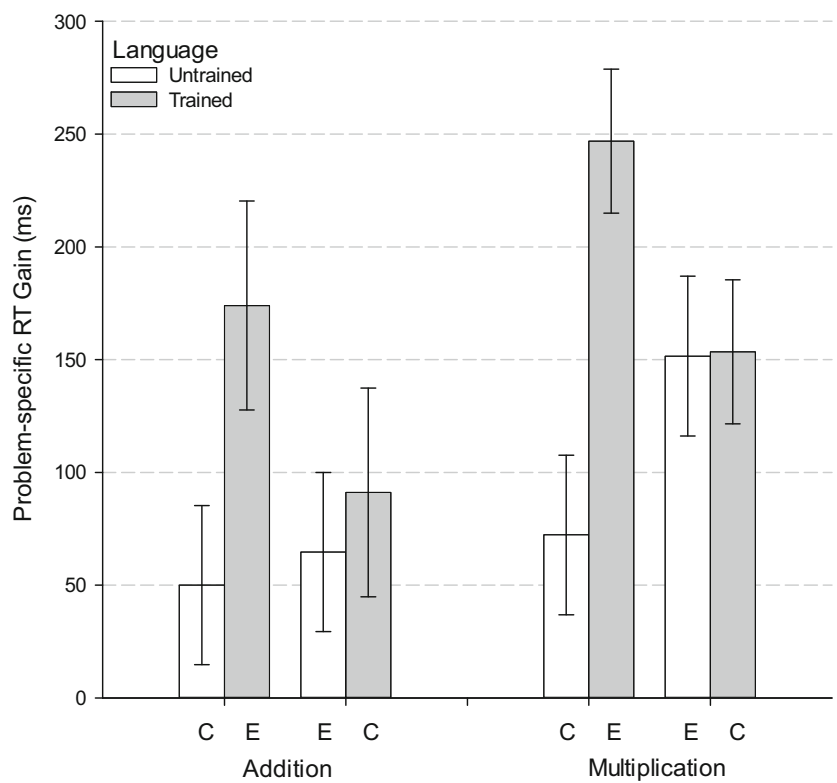

Fig. 2 Mean problem-specific response time (RT) gain in $\mathrm{ms}$ in the test phase. $\mathrm{C}=$ test language Chinese. $\mathrm{E}=$ test language English. Error bars are \pm 1 standard error

\section{Effects of operation on transfer within training language}

English trained A Group (i.e., addition vs. multiplication when trained in English) $\times$ Language Type (test language trained vs. untrained) indicated only a significant effect of Language Type on RT gains $[F(1,30)=17.7, \mathrm{MSE}=20$, $\left.089, \eta_{\mathrm{P}}^{2}=0.37, p<0.001\right]$, reflecting larger RT gains in the trained English language $[211 \mathrm{~ms}, t(31)=6.03, p<.00001, \mathrm{SE}$ $=35.0$ relative to 0 gains] compared to the untrained Chinese language [61 ms, $t(31)=3.43, p=.002, \mathrm{SE}=17.9]$. There were no effects involving the operation factor when English was the trained language (all $p \mathrm{~s}>0.28$ ).

Chinese trained The corresponding Group (addition vs. multiplication) $\times$ Language Type ANOVA of RT gains with Chinese trained and English untrained indicated no significant effects (all $p>.06$ ). Mean RT gains were $122 \mathrm{~ms}$ in the trained Chinese language $[t(31)=6.44, p<.00001, \mathrm{SE}=19.0]$ and $108 \mathrm{~ms}$ in the untrained English language $[t(31)=3.55, p=$ $.001, \mathrm{SE}=30.5]$. Thus, unlike when English was the trained language, there was no evidence that RT gains differed between languages when Chinese was trained $[F(1,30)=0.14$, $\mathrm{MSE}=17,750, \eta_{\mathrm{P}}^{2}=0.005, p=.72$ for the interaction].

\section{Discussion}

The present experiment tested the theory that Chinese-English bilinguals may develop language-specific memory representations for everyday simple addition and multiplication facts. This possibility was supported previously by evidence that retrieval-induced interference of addition memory by retrieval practice of multiplication counterparts was observed only when the practice and test languages were the same and not when the languages differed (Campbell \& Dowd, 2012; see also Campbell \& Epp, 2004). Such language-specificity of memory for arithmetic facts is consistent with evidence that training bilinguals on new multi-digit arithmetic problems 
produced RT gains in the trained language that did not generalize to the untrained language (Dehaene et al., 1999; Spelke \& Tsivkin, 2001). Here we adopted a similar paradigm to that used by Dehaene et al. that afforded measurement of language specificity of training in Chinese-English bilinguals' singledigit addition and multiplication.

For addition, the results indicated that RT gains from training in one language provided little, if any, benefit to the trained problems tested in the untrained language. In contrast, when multiplication was trained in Chinese, problem-specific RT gains were equivalent for the trained Chinese and untrained English language. In other words, there was nearly complete transfer of learning of the Chinese trained problems to the untrained English language. This result is consistent with two main alternative potential explanations. First, crosslanguage transfer would occur if participants mentally recoded the English problems into verbal Chinese or visual Arabic stimuli and then retrieved the solution in Chinese and translated the answer back into English for response output. This Chinese-based translation strategy would take advantage of problem-specific RT gains from training in Chinese because the nominally English-language trials would be mediated by the trained Chinese language. This would allow much of the language-specific RT gains from Chinese practice to transfer to the untrained English language. A second possible explanation is that arithmetic memory for both languages is mediated by a common abstract or amodal representational format (McCloskey \& Macaruso, 1995; see Campbell, 2015; Cohen Kadosh \& Walsh, 2009). In this case Chinese practice would strengthen the common abstract representation and this learning would transfer to English because English performance would be mediated by the strengthened abstract representation.

An explanation for the observed generalization of training from Chinese to English based on translation (i.e., for the test phase in English after training in Chinese, translate to Chinese then back to English) is consistent with the present results. In contrast, the proposal of a common amodal representation is difficult to reconcile with the results. The latter theory implies that cross-language transfer should be at least approximately symmetrical because much of the RT gains would be associated with strengthening the common representation (Rickard \& Bourne, 1996). Consequently, training in either language should produce substantial positive transfer of learning to the other. In fact, however, when English was trained, problemspecific RT gains were much greater in English compared to the gains observed in the untrained Chinese language. Specifically, gains in the trained English language were $211 \mathrm{~ms}$ (averaging across operations) compared to gains of $61 \mathrm{~ms}$ in the untrained Chinese language. When training occurred in Chinese, there was little difference in the RT gains in the trained Chinese language $(122 \mathrm{~ms})$ and the untrained English language (108 ms). In other words, there was a strong asymmetry between Chinese and English in the languagespecificity of RT gains, a result that is at odds with the proposal of a common abstract or amodal memory system for arithmetic facts.

Instead the results suggest that after Chinese training, English arithmetic (particularly multiplication) was mediated by translation to Chinese, whereas, in contrast, Chinese translation was used minimally during training in English, and consequently there were minimal gains in the untrained Chinese language during the test phase. Moreover, the results indicate that the problem-specific English RT gains from English training must, at least in part, arise from languagespecific learning. RT gains for English when it was the trained language were greater than the gains for Chinese when it was the trained language $[211 \mathrm{~ms}$ vs. $122 \mathrm{~ms}, t(31)=2.46, \mathrm{SE}=$ $36.0, p=.020]$; thus, the gains for English were greater than would be expected even if the full benefits of training in Chinese were transferred to English. In other words, the gains were too large to be explained by Chinese-language mediation and provide evidence of language-specific RT gains for specific problems. These gains evidently were not sufficient, however, to induce participants to use English-language trained arithmetic facts for translation purposes when Chinese was required at test; instead, they continued to rely on their already-fluent Chinese-language process for untrained problems in Chinese.

The generalization results suggest that training multiplication in Chinese promoted a translation strategy for multiplication in the untrained English language, but there was little evidence that training addition in Chinese promoted translation for English addition. Campbell and Epp (2004) suggested that English-to-Chinese translation was more likely for multiplication than addition, possibly because multiplication memory is usually weaker (e.g., Campbell \& Xue, 2001), regardless of language, and individuals do not develop sufficient memory strength in English to rely on English-language representations. In contrast, memory strength for addition in English might often be sufficient to support direct retrieval in English. Such an asymmetry in English-to-Chinese translation (i.e., more likely for multiplication than addition) would weaken cross-language gains for addition when Chinese was the trained language.

\section{Conclusions}

The results provided evidence of language-specific strengthening of memory for everyday arithmetic facts. We cannot tell from the current data, however, whether the participants possessed language-specific memories for arithmetic prior to the experiment, or acquired them from the language-specific training they received in the experiment. Given that it is possible to acquire language-specific arithmetic memories, then 
the natural development of arithmetic fact networks in multiple languages would depend on the opportunities for strengthening memories for the facts in each language. The present results suggest that a strong asymmetry in arithmetic-fact memory strength can promote a translation strategy based on the stronger language. In this case, arithmetic facts stored and routinely used in two languages would be more likely in bilinguals whose arithmetic experiences in each language were acquired simultaneously and become approximately equal in strength.

Author note This research was supported by a grant from the Natural Sciences and Engineering Research Council of Canada to Jamie Campbell.

\section{References}

Butterworth, B., \& Reeve, R. (2008). Verbal counting and spatial strategies in numerical tasks: Evidence from Indigenous Australia. Philosophical Psychology, 21, 443-457.

Butterworth, B., Reeve, R., \& Reynolds, F. (2011). Using mental representations of space when words are unavailable: Studies of Enumeration and Arithmetic in Indigenous Australia. Journal of Cross-Cultural Psychology, 42, 630-638.

Campbell, J. I. D. (2015). How abstract is arithmetic? In A. Dowker \& R. Cohen Kadosh (Eds.), Oxford Handbook of Numerical Cognition (pp. 140-157). Oxford: Oxford University Press.

Campbell, J. I. D., \& Dowd, R. (2012). Inter-operation transfer in Chinese-English bilinguals' arithmetic. Psychonomic Bulletin \& Review, 19, 948-954.

Campbell, J. I. D., \& Epp, L. (2004). An encoding-complex approach to numerical cognition in Chinese-English bilinguals. Canadian Journal of Experimental Psychology, 58, 229-244.

Campbell, J. I. D., \& Therriault, N. (2013). Retrieval-induced forgetting of arithmetic facts but not rules. Journal of Cognitive Psychology, $25,717-724$
Campbell, J. I. D., \& Xue, Q. (2001). Cognitive arithmetic across cultures. Journal of Experimental Psychology: General, 130, 299-315.

Cohen Kadosh, R., \& Walsh, V. (2009). Numerical representation in the parietal lobes: Abstract or not abstract? Behavioral and Brain Sciences, 32, 313-328. doi:10.1017/S0140525X09990938

Cohen, L., Dehaene, S., Chochon, F., Lehericy, S., \& Naccache, L. (2000). Language and calculation within the parietal lobe: A combined cognitive, anatomical, and fMRI study. Neuropsychologia, 38, 1426-1440.

Dehaene, S., Spelke, E., Pinal, P., Stanescu, R., \& Tsivkin, S. (1999). Sources of mathematical thinking: Behavioral and brain-imaging evidence. Science, 284, 970-974.

Everett, C., \& Madora, K. (2012). Quantity recognition among speakers of an anumeric language. Cognitive Science, 36, 130-141.

Frank, M., Everett, D., Fedorenko, E., \& Gibson, E. (2008). Number as a cognitive technology: Evidence from Piraha language and cognition. Cognition, 108, 819-824.

Gelman, R., \& Butterworth, B. (2005). Number and language: How are they related? Trends in Cognitive Science, 9, 6-10.

McCloskey, M., \& Macaruso, P. (1995). Representing and using numerical information. American Psychologist, 50, 351-363.

Pica, P., Lemer, C., Izard, V., \& Dehaene, S. (2004). Exact and approximate arithmetic in an Amazonian indigene group. Science, 306, 499-503. doi:10.1126/science.1102085

Rickard, T. C., \& Bourne, L. E., Jr. (1996). Some tests of an identical elements model of basic arithmetic skills. Journal of Experimental Psychology: Learning, Memory, \& Cognition, 22, 1281-1295.

Rusconi, E., Galfano, G., \& Job, R. (2007). Bilingualism and cognitive arithmetic. In I. Kecskes \& L. Albertazzi (Eds.), Cognitive aspects of bilingualism (pp. 153-174). New York: Springer.

Salillas, E., \& Carreiras, M. (2014). Core number representations are shaped by language. Cortex, 52, 1-11. doi:10.1016/j.cortex.2013. 12.009

Spaepen, E., Coppola, M., Spelke, E., Carey, S., \& Goldin-Meadow, S. (2011). Number without a language model. Proceedings of the National Academy of Sciences, 108, 3163-3168.

Spelke, E. S., \& Tsivkin, S. (2001). Language and number: A bilingual training study. Cognition, 78, 45-88.

Venkatraman, V., Siong, A. C., Chee, M. W. L., \& Ansari, D. (2006). Effect of language switching on arithmetic: A bilingual fMRI study. Journal of Cognitive Neuroscience, 18, 64-74. 